\title{
18. Spontaneous Diabetes Produced by Selective Breeding of Normal Wistar Rats
}

\author{
By Yoshio Goto, Masaei KakizaKI, and Naoyoshi Masaki \\ The Third Department of Internal Medicine, Hirosaki University \\ School of Medicine, Hirosaki, Japan \\ (Comm. by Toshio Kurokawa, M. J. A., Jan. 13, 1975)
}

Diabetes mellitus is thought of as a disease of inheritant nature. The mode of transmission, however, is still inconclusive. An autosomal recessive trait theory was postulated by Pincus and White ${ }^{1)}$ and supported by Steinberg. ${ }^{2)}$ On the other hand, Cammidge ${ }^{3)}$ and Stimmler and Elliott ${ }^{4}$ thought that juvenile onset type was recessive and adult onset type was dominant, and recently a multifactorial inheritance theory has been proposed by Simpson.5)

In 1968, Craighead and McLane ${ }^{6)}$ observed that an inoculation of a variant of encephalomyocarditis virus produced a diabetic state in mice and found that the islets of their pancreas were selectively damaged. This experiment suggested a possibility that diabetes might be a disease of viral infection. If a family has less resistance to the virus, a higher incidence of diabetes would occur in this family and the phenomenon of a higher prevalance of the disease in certain family will resemble an inheritance, just as tuberculosis was thought to be a disease of inheritant nature in the early days. Therefore, a question will arise: is it certainly true that diabetes is a disease of inheritance?

From this background of our present knowledge, we cristalized the following working hypothesis. If animals, selected from normal population, with a glucose tolerance test (GTT) slightly deviated from normal range, are mated, their offsprings may have a more impaired GTT than the parents. If it is so, the repeat of this procedure over several generations would finally produce diabetic animals. If it is not so, diabetic state would be produced only by definite gene (s) unless the occurrence of mutation, or by other factors, such as viral infection, etc. The present study was carried out according to this hypothesis.

Methods. One hundred and thirty male rats and 81 female rats of Wistar strain, supplied from the Nogawa Farm of Japan Clea Co., Kawasaki, were used as foundation stock (P). The oral GTT was performed in the rats, weighing 150-200 $\mathrm{g}$ and fed Oriental Chow (MF) ad libitum, after 12 hours fasting and the rats with slight 
glucose intolerance were selected as breeding stock. GTT was made as following: $20 \%$ glucose solution $(2 \mathrm{~g} / \mathrm{kg})$ was administered orally by metal catheter attached with $5 \mathrm{ml}$ size syringe and $0.05 \mathrm{ml}$ of blood specimens were collected from tail vessels by cutting its end, prior to, and 30, 60, 90 and 120 minutes after the glucose administration. Urine sugar was tested with Tes-Tape when urinated. Blood glucose was determined by glucose-oxidase method using Glucostat.

Of the 211 foundation stock rats, 9 male and 9 female rats were selected as breeding stock. For breeding, male and female rats were housed together in a cage for 5-10 days. Pregnant rats were housed in an individual delivery-cage in which rice straw were put in. When infant rats were grown up to approximately $80 \mathrm{~g}$ of body weight, they were taken out of the delivery-cage and housed in cages separately by sex. By the breeding, 162 offsprings $\left(F_{1}\right)$ were obtained, i.e., 89 male and 73 female rats. GTT was made in these offsprings when their body weight was 150-180 g. According to the result of the test, 12 male and 13 female rats were finally selected as breeding stock and the crossing was made as the same way described above. Two hundred and four offsprings $\left(\mathrm{F}_{2}\right)$, i.e., 116 male and 88 female rats were obtained. The same procedure was repeated in this generation.

For the selection of the breeding stock rats, the second GTT was repeated within two weeks in the rats screened by the (first) test and the rats with two impaired tests were chosen. In this experiment, inbreeding, especially sib-breeding was avoided. The grade of glucose intolerance was expressed as the sum of blood glucose values

Table I. Mean glucose tolerance tests of the foundation stock rats, the first and the second generation

\begin{tabular}{|c|c|c|c|c|c|c|c|}
\hline \multirow{2}{*}{$\begin{array}{l}\text { Gen- } \\
\text { era- } \\
\text { tion }\end{array}$} & \multirow{2}{*}{$\begin{array}{l}\text { Sex } \\
\text { No. }\end{array}$} & \multicolumn{6}{|c|}{ Blood glucose (mg/dl) } \\
\hline & & Fast & 30 & 60 & 90 & 120 & Sum \\
\hline \multirow{2}{*}{$\mathrm{P}$} & M 130 & $\begin{array}{l}79.0 \\
\pm 12.0\end{array}$ & $\begin{array}{l}139.4 \\
\quad \pm 19.0\end{array}$ & $\begin{array}{l}131.5 \\
\quad \pm 18.4\end{array}$ & $\begin{array}{l}107.6 \\
\quad \pm 16.5\end{array}$ & $\begin{array}{l}95.4 \\
\quad \pm 12.5\end{array}$ & $\begin{array}{l}552.8 \\
\quad \pm 57.3\end{array}$ \\
\hline & F 81 & $\begin{array}{l}81.2 \\
\pm 11.7\end{array}$ & $\begin{array}{l}142.2 \\
\quad \pm 17.8\end{array}$ & $\begin{array}{l}129.8 \\
\quad \pm 18.5\end{array}$ & $\begin{array}{l}112.3 \\
\quad \pm 15.5\end{array}$ & $\begin{array}{l}103.5 \\
\quad \pm 13.5\end{array}$ & $\begin{array}{l}568.4 \\
\quad \pm 61.1\end{array}$ \\
\hline \multirow{2}{*}{$\mathrm{F}_{1}$} & M 89 & $\begin{array}{l}96.5 \\
\quad \pm 12.9\end{array}$ & $\begin{array}{l}167.2 \\
\quad \pm 25.6\end{array}$ & $\begin{array}{l}149.1 \\
\quad \pm 21.2\end{array}$ & $\begin{array}{l}126.7 \\
\quad \pm 14.6\end{array}$ & $\begin{array}{l}112.2 \\
\quad \pm 11.4\end{array}$ & $\begin{array}{l}649.6 \\
\quad \pm 61.1\end{array}$ \\
\hline & F 73 & $\begin{array}{l}93.5 \\
\quad \pm 9.5\end{array}$ & $\begin{array}{l}157.6 \\
\quad \pm 23.0\end{array}$ & $\begin{array}{l}136.6 \\
\quad \pm 19.0\end{array}$ & $\begin{array}{l}121.2 \\
\quad \pm 12.9\end{array}$ & $\begin{array}{l}115.8 \\
\quad \pm 13.9\end{array}$ & $\begin{array}{l}624.8 \\
\quad \pm 63.3\end{array}$ \\
\hline \multirow{2}{*}{$\mathbf{F}_{2}$} & & $\begin{array}{l}103.3 \\
\quad \pm 15.9\end{array}$ & $\begin{array}{l}192.6 \\
\quad \pm 32.9\end{array}$ & $\begin{array}{l}162.8 \\
\quad \pm 27.3\end{array}$ & $\begin{array}{l}137.9 \\
\quad \pm 21.3\end{array}$ & $\begin{array}{r}123.7 \\
\quad \pm 17.8\end{array}$ & $\begin{array}{l}719.8 \\
\quad \pm 93.1\end{array}$ \\
\hline & $\mathrm{F} \quad 88$ & $\begin{array}{l}102.3 \\
\quad \pm 17.0\end{array}$ & $\begin{array}{l}180.7 \\
\quad \pm 29.4\end{array}$ & $\begin{array}{l}152.6 \\
\quad \pm 33.6\end{array}$ & $\begin{array}{l}136.8 \\
\quad \pm 29.2\end{array}$ & $\begin{array}{l}126.6 \\
\quad \pm 27.2\end{array}$ & $\begin{array}{l}698.9 \\
\quad \pm 113.0\end{array}$ \\
\hline
\end{tabular}

Mean \pm S. D. 
Table II. Combination of mating in the foundation stock rats and sum of blood glucose of the offsprings $\left(\mathrm{F}_{1}\right)$

\begin{tabular}{|c|c|c|c|c|}
\hline \multirow{3}{*}{ No. } & \multicolumn{2}{|r|}{ Breeding stock } & \multicolumn{2}{|r|}{ Offspring $\left(\mathrm{F}_{1}\right)$} \\
\hline & \multirow{2}{*}{ Sex } & Glucose tolerance test & \multirow{2}{*}{ Sex } & \multirow{2}{*}{ Sum of blood glucose } \\
\hline & & Fast. $30 \quad 60 \quad 90 \quad 120$ sum & & \\
\hline 1 & $\begin{array}{l}\mathrm{M} \\
\mathrm{F}\end{array}$ & $\begin{array}{llllll}87 & 177 & 180 & 163 & 131 & 738 \\
87 & 148 & 159 & 136 & 114 & 644\end{array}$ & $\frac{\mathrm{M}}{\mathrm{F}}$ & $\begin{array}{l}789,780,752,778 \\
793,794,712,742,730\end{array}$ \\
\hline 2 & $\mathrm{M}$ & $\begin{array}{llllll}92 & 148 & 162 & 144 & 109 & 655 \\
93 & 162 & 127 & 114 & 101 & 597\end{array}$ & & $\begin{array}{l}599,587,572,665,506,645^{*} \\
548\end{array}$ \\
\hline 3 & $\begin{array}{l}\mathrm{M} \\
\mathrm{F}\end{array}$ & $\begin{array}{llllll}107 & 196 & 169 & 137 & 119 & 728 \\
103 & 178 & 140 & 126 & 111 & 658\end{array}$ & $\begin{array}{l}\mathrm{M} \\
\mathrm{F}\end{array}$ & $\begin{array}{l}594,627,632,600,615,615,590 \\
602,604,656,629,639,715\end{array}$ \\
\hline 4 & $\begin{array}{l}\mathrm{M} \\
\mathrm{F}\end{array}$ & $\begin{array}{llllll}87 & 194 & 169 & 124 & 119 & 693 \\
87 & 184 & 152 & 129 & 115 & 667\end{array}$ & $\mathrm{M}$ & $\begin{array}{l}627,756,657,648,595 \\
557,652,615\end{array}$ \\
\hline 5 & $\begin{array}{l}\mathrm{M} \\
\mathrm{F}\end{array}$ & $\begin{array}{llllll}96 & 182 & 137 & 113 & 103 & 631 \\
97 & 128 & 148 & 137 & 127 & 637\end{array}$ & $\begin{array}{l}\mathrm{M} \\
\mathrm{F}\end{array}$ & $\begin{array}{l}745,694,713 \\
689,595,662,690\end{array}$ \\
\hline 6 & $\begin{array}{l}\mathrm{M} \\
\mathrm{F}\end{array}$ & $\begin{array}{rrrrrr}100 & 148 & 157 & 153 & 125 & 683 \\
90 & 140 & 150 & 131 & 122 & 633\end{array}$ & $\begin{array}{l}\mathrm{M} \\
\mathrm{F}\end{array}$ & $\begin{array}{l}729,704,757,623 \\
617,709,619,552,629,608^{*}, 569^{*}, 573^{*}\end{array}$ \\
\hline 7 & $\begin{array}{l}\mathrm{M} \\
\mathrm{F}\end{array}$ & $\begin{array}{llllll}72 & 157 & 154 & 127 & 111 & 621 \\
90 & 147 & 169 & 129 & 116 & 651\end{array}$ & $\begin{array}{l}\mathrm{M} \\
\mathrm{F}\end{array}$ & $\begin{array}{l}599,688,679,685,591,618,701,627,662 \\
\text { none } \\
574,662^{*}\end{array}$ \\
\hline 8 & $\begin{array}{l}\mathrm{M} \\
\mathrm{F}\end{array}$ & $\begin{array}{llllll}72 & 176 & 180 & 154 & 127 & 709 \\
94 & 153 & 126 & 113 & 102 & 588\end{array}$ & $\mathrm{M}$ & $\begin{array}{l}636,680,675,743^{*}, 654^{*}, 555^{*}, 632^{*} \\
658,588,585,568,543^{*}\end{array}$ \\
\hline 9 & $\begin{array}{l}\mathrm{M} \\
\mathrm{F}\end{array}$ & $\begin{array}{llllll}74 & 162 & 240 & 123 & 101 & 700 \\
84 & 150 & 159 & 146 & 143 & 682\end{array}$ & $\stackrel{\mathrm{M}}{\mathrm{F}}$ & $\begin{array}{l}565,559,570,614,578,562 \\
567,577,556,573\end{array}$ \\
\hline 10 & $\begin{array}{l}\mathrm{M} \\
\mathrm{F}\end{array}$ & $\begin{array}{llllll}92 & 148 & 162 & 144 & 109 & 655 \\
87 & 184 & 152 & 129 & 115 & 667\end{array}$ & $\begin{array}{l}\mathrm{M} \\
\mathrm{F}\end{array}$ & $\begin{array}{l}620,636,614,599 \\
605,583,555,592\end{array}$ \\
\hline 11 & $\begin{array}{l}\mathrm{M} \\
\mathrm{F}\end{array}$ & $\begin{array}{rrrrrr}107 & 196 & 169 & 137 & 119 & 728 \\
97 & 128 & 148 & 137 & 127 & 637\end{array}$ & $\mathrm{M}$ & $\begin{array}{l}637,653,557,665,742 \\
586,628,574,637,603,643^{*}\end{array}$ \\
\hline 12 & $\begin{array}{l}\mathrm{M} \\
\mathrm{F}\end{array}$ & $\begin{array}{llllll}92 & 148 & 162 & 144 & 109 & 655 \\
94 & 153 & 126 & 113 & 102 & 588\end{array}$ & $\mathrm{M}$ & $\begin{array}{l}628,632,583,712,675,682,606 \\
673,682,643\end{array}$ \\
\hline 13 & $\begin{array}{l}\mathrm{M} \\
\mathrm{F}\end{array}$ & $\begin{array}{llllll}87 & 177 & 180 & 163 & 131 & 738 \\
90 & 140 & 150 & 131 & 122 & 633\end{array}$ & $\begin{array}{l}\mathrm{M} \\
\mathrm{F}\end{array}$ & $\begin{array}{l}656,689,694,635^{*} \\
692,638,760,624,745,642\end{array}$ \\
\hline 14 & $\begin{array}{l}\mathrm{M} \\
\mathrm{F}\end{array}$ & $\begin{array}{rrrrrr}107 & 196 & 169 & 137 & 119 & 728 \\
87 & 142 & 137 & 128 & 116 & 610\end{array}$ & & $\begin{array}{l}699,683,736,674,708,659 \\
584,586,587,689,676\end{array}$ \\
\hline 15 & $\begin{array}{l}\mathrm{M} \\
\mathrm{F}\end{array}$ & $\begin{array}{llllll}100 & 148 & 157 & 153 & 125 & 683 \\
103 & 178 & 140 & 126 & 111 & 658\end{array}$ & $\frac{\mathrm{M}}{\mathrm{F}}$ & $\begin{array}{l}556,552,606,603,669 \\
594,543,590,562,576,552^{*}, 579,583,506,559^{*}\end{array}$ \\
\hline 16 & $\begin{array}{l}\mathrm{M} \\
\mathrm{F}\end{array}$ & $\begin{array}{llllll}72 & 157 & 154 & 127 & 111 & 621 \\
84 & 150 & 159 & 146 & 143 & 682\end{array}$ & $\begin{array}{l}\mathrm{M} \\
\mathrm{F}\end{array}$ & $\begin{array}{l}691,753,698,635,672 \\
711,679,633\end{array}$ \\
\hline
\end{tabular}

Bold type indicates cases with a positive test for urine sugar.

Asterisk represents cases of retarded growth.

at five time periods, i.e., fasting, 30, 60, 90 and 120 minutes.

Results. The mean GTT of the foundation stock $(\mathrm{P})$, of the first generation $\left(F_{1}\right)$ and of the second generation $\left(F_{2}\right)$ are shown in Table I. The mean blood glucose value becomes high with generation at each time period and the values of $F_{1}$ and $F_{2}$ are significantly higher in comparison with those of $\mathrm{P}$. The combination of crossing and the sum of blood glucose of the offsprings are shown in Tables II and III. Cases with a positive test for urine sugar were shown in bold type. There were, however, many cases in which urine sugar was not tested 
Table III. Combination of mating in the first generation rats and sum of blood glucose of the offsprings $\left(\mathrm{F}_{2}\right)$

\begin{tabular}{|c|c|c|c|c|}
\hline \multirow{3}{*}{ No. } & \multicolumn{2}{|r|}{ Breeding stock $\left(\mathrm{F}_{1}\right)$} & \multicolumn{2}{|r|}{ Offspring $\left(\mathrm{F}_{2}\right)$} \\
\hline & \multirow{2}{*}{ Sex } & Glucose tolerance test & \multirow{2}{*}{ Sex } & \multirow{2}{*}{ Sum of blood glucose } \\
\hline & & Fast. $30 \quad 60 \quad 90120$ sum & & \\
\hline 1 & $\stackrel{\mathrm{M}}{\mathrm{F}}$ & $\begin{array}{llllll}111 & 234 & 163 & 142 & 139 & 789 \\
104 & 163 & 136 & 131 & 128 & 662\end{array}$ & $\mathrm{M}$ & $\begin{array}{l}674,918,804,726,728,782 \\
760,713,689\end{array}$ \\
\hline 2 & $\begin{array}{l}\mathrm{M} \\
\mathrm{F}\end{array}$ & $\begin{array}{llllll}104 & 225 & 161 & 146 & 121 & 757 \\
115 & 211 & 181 & 146 & 141 & 794\end{array}$ & $\stackrel{\mathrm{M}}{\mathrm{F}} \varepsilon$ & $\begin{array}{l}603,751,697,686,838,650 \\
861, \mathbf{6 2 8}, \mathbf{6 7 6}, 656\end{array}$ \\
\hline 3 & $\frac{\mathrm{M}}{\mathrm{F}}$ & $\begin{array}{llllll}92 & 180 & 176 & 134 & 119 & 701 \\
99 & 217 & 157 & 137 & 132 & 742\end{array}$ & $\begin{array}{l}\mathrm{M} \\
\mathrm{F}\end{array}$ & $\begin{array}{l}608,741,679,735,677 \\
609,664, \quad 1005,1090,806,738,823,890\end{array}$ \\
\hline 4 & $\begin{array}{l}\mathrm{M} \\
\mathrm{F}\end{array}$ & $\begin{array}{rlllll}122 & 201 & 149 & 138 & 135 & 745 \\
86 & 207 & 160 & 139 & 117 & 709\end{array}$ & $\begin{array}{l}\mathrm{M} \\
\mathrm{F}\end{array}$ & $\begin{array}{l}877,900,754,665,650,882,847,950,1040 \\
974,694,683,903\end{array}$ \\
\hline 5 & $\frac{\mathrm{M}}{\mathrm{F}}$ & $\begin{array}{rrrrrr}95 & 163 & 148 & 138 & 118 & 662 \\
103 & 173 & 132 & 129 & 121 & 658\end{array}$ & $\begin{array}{l}\mathrm{M} \\
\mathrm{F}\end{array}$ & $\begin{array}{l}591,560,646,602,609,644,685,590 \\
684,607,675\end{array}$ \\
\hline 6 & $\frac{\mathrm{M}}{\mathrm{F}}$ & $\begin{array}{rrrrrr}80 & 164 & 166 & 133 & 119 & 662 \\
102 & 231 & 180 & 144 & 136 & 793\end{array}$ & $\stackrel{\mathrm{M}}{\mathrm{F}} 7$ & $\begin{array}{l}847,799,824,734,817,865,861,786,775 \\
702,713,622,710\end{array}$ \\
\hline 7 & $\begin{array}{l}\mathrm{M} \\
\mathrm{F}\end{array}$ & $\begin{array}{rrrrrr}98 & 138 & 134 & 141 & 125 & 636 \\
103 & 169 & 167 & 142 & 130 & 711\end{array}$ & $\begin{array}{l}\mathrm{M} \\
\mathrm{F}\end{array}$ & $\begin{array}{l}758 \\
682\end{array}$ \\
\hline 8 & $\mathrm{M}$ & $\begin{array}{rrrrrr}109 & 194 & 148 & 128 & 115 & 694 \\
99 & 154 & 139 & 140 & 147 & 679\end{array}$ & $\mathrm{M} 7$ & $\begin{array}{l}733,649,651,661,705^{*} \\
\mathbf{6 9 0}, \mathbf{6 9 6}, \mathbf{7 4 9}, \mathbf{6 9 1}, 612^{*}\end{array}$ \\
\hline 9 & $\mathrm{M}$ & $\begin{array}{rrrrrr}94 & 165 & 187 & 129 & 123 & 698 \\
106 & 170 & 154 & 135 & 124 & 689\end{array}$ & $\begin{array}{l}\mathrm{M} \\
\mathrm{F}\end{array}$ & $\begin{array}{l}665,625,712,608,588 \\
593,644,653,630,577^{*}, 977^{*}\end{array}$ \\
\hline 10 & $\mathrm{M}$ & $\begin{array}{rrrrrr}92 & 180 & 176 & 134 & 119 & 701 \\
115 & 211 & 181 & 146 & 141 & 794\end{array}$ & $\underset{\mathrm{F}}{\mathrm{M}} 6$ & $\begin{array}{l}782,885,734,699,648,586,663,753 \\
630,645,641,714,632\end{array}$ \\
\hline 11 & $\stackrel{\mathrm{M}}{\mathrm{F}}$ & $\begin{array}{rlllll}122 & 201 & 149 & 138 & 135 & 745 \\
99 & 217 & 157 & 137 & 132 & 742\end{array}$ & $\stackrel{\mathrm{M}}{\mathrm{F}} \mathrm{g}$ & $\begin{array}{l}626,897^{*}, \mathbf{7 9 6}, 932,748,768 \\
\mathbf{9 0 7 *}, \mathbf{7 6 0} * \mathbf{8 6 4}, \mathbf{7 4 3}, 603,611^{*}, 866\end{array}$ \\
\hline 12 & $\begin{array}{l}\mathrm{M} \\
\mathrm{F}\end{array}$ & $\begin{array}{rlllll}104 & 225 & 161 & 146 & 121 & 757 \\
86 & 207 & 160 & 139 & 117 & 709\end{array}$ & $\begin{array}{l}\mathrm{M} \\
\mathrm{F}\end{array}$ & $\begin{array}{l}670, \mathbf{7 7 9}, 856,747,706,913 *, 693,644 \\
574,569,637,724,623,1084^{*}\end{array}$ \\
\hline 13 & $\underset{\mathrm{M}}{\mathrm{M}}$ & $\begin{array}{rrrrrr}95 & 163 & 148 & 138 & 118 & 662 \\
103 & 173 & 132 & 129 & 121 & 658\end{array}$ & $\begin{array}{l}\mathrm{M} \\
\mathrm{F}\end{array}$ & $\begin{array}{l}693,617,675,692,704,779,703,678,650 \\
664, \mathbf{6 5 6 , 5 8 4}\end{array}$ \\
\hline 14 & $\frac{\mathrm{M}}{\mathrm{F}}$ & \begin{tabular}{rrrrrr|}
80 & 164 & 166 & 133 & 119 & 662 \\
102 & 231 & 180 & 144 & 136 & 793
\end{tabular} & $\begin{array}{l}\text { M } \\
\text { F } 6\end{array}$ & $\begin{array}{l}\mathbf{7 2 5}, 848,705.706,694 \\
\mathbf{6 8 7}, 694,713,713,715, \mathbf{6 2 9} *, \mathbf{6 5 7} *, 690 *\end{array}$ \\
\hline 15 & $\mathrm{M}$ & $\begin{array}{rlllll}117 & 145 & 141 & 130 & 123 & 656 \\
87 & 129 & 136 & 123 & 115 & 590\end{array}$ & $\begin{array}{ll}\mathrm{M} & 6 \\
\mathrm{~F} & 6\end{array}$ & $\begin{array}{l}651,663,568,669 \\
634,601,585,637^{*}, 653,601,608\end{array}$ \\
\hline 16 & $\frac{\mathrm{M}}{\mathrm{F}}$ & $\begin{array}{llllll}98 & 138 & 134 & 141 & 125 & 636 \\
91 & 186 & 151 & 127 & 137 & 692\end{array}$ & $\begin{array}{ll}\mathrm{M} & 6 \\
\mathrm{~F} & 6\end{array}$ & $\begin{array}{l}683,697,602,690,723,668,646,726 \\
686,801,715,609^{*}\end{array}$ \\
\hline 17 & $\mathrm{M}$ & $\begin{array}{llllll}100 & 152 & 162 & 124 & 115 & 653 \\
110 & 195 & 160 & 145 & 150 & 760\end{array}$ & $\begin{array}{ll}\mathrm{M} & 6 \\
\mathrm{~F} & 6\end{array}$ & $\begin{array}{l}624,638,719,673,667,647^{*} \\
619,557,613\end{array}$ \\
\hline 18 & $\mathrm{M}$ & $\begin{array}{llllll}84 & 163 & 155 & 146 & 121 & 669 \\
95 & 188 & 163 & 151 & 148 & 745\end{array}$ & $\begin{array}{l}\mathrm{M} \\
\mathrm{F}\end{array}$ & $\begin{array}{l}660,859,697, \mathbf{7 6 0}, 661,840 \\
713, \mathbf{6 4 4}, 622,576, \mathbf{6 8 8}, \mathbf{6 4 3}\end{array}$ \\
\hline
\end{tabular}

Bold type indicates cases with a positive test for urine sugar.

Asterisk represents cases of retarded growth.

because of no urination during the test. In each group of litters, there were few rats of retarded growth and these rats are shown as asterisk in the tables. There was no special pattern of GTT in these rats.

Distributions of the sum of blood glucose in each generation are shown in Fig. 1. It is easy to understand in this figure that the dis- 


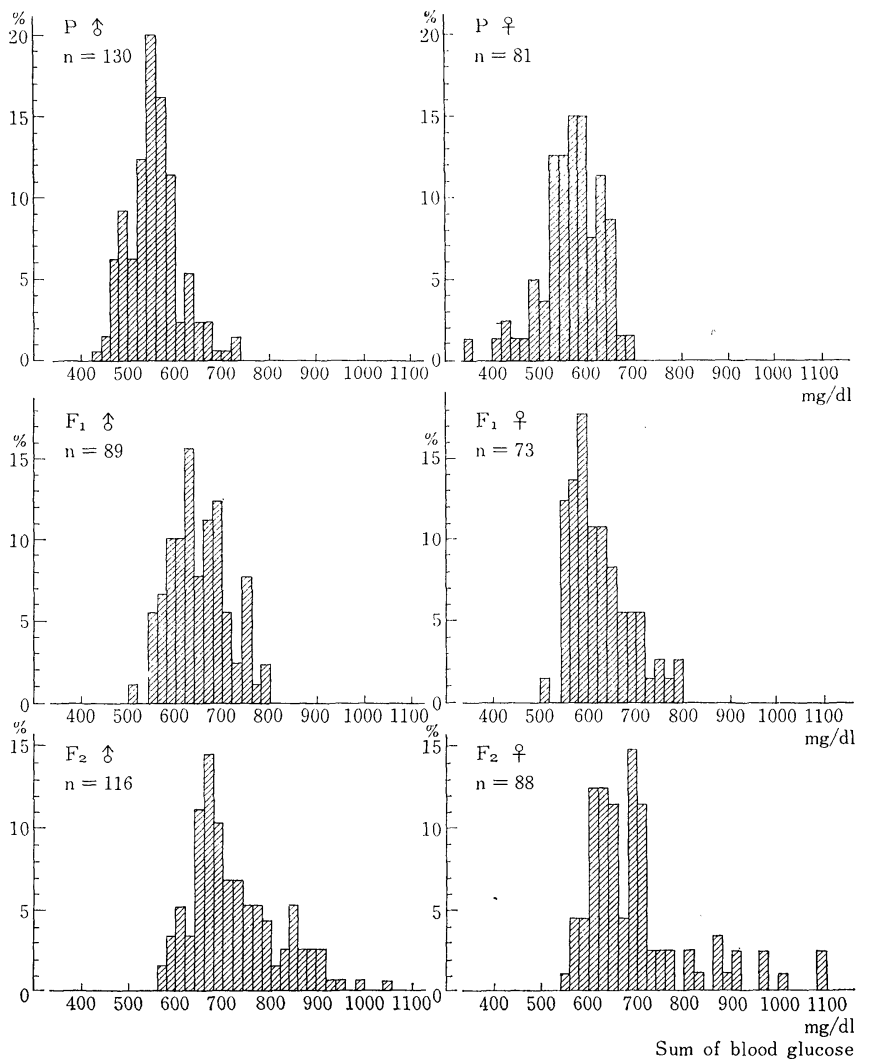

Fig. 1. Distribution of sum of blood glucose in each generation.

tribution curves shift to the right with the advance of the generation. This indicates that the selective breeding of the rats with a GTT slightly deviated from normal range yields rats with a test more deviated from normal. Some of $\mathrm{F}_{2}$ had a typical diabetic GTT with a two hour value exceeding $200 \mathrm{mg} / \mathrm{dl}$ and a positive test for urine sugar. There was no rats in $F_{1}$ which sum of glucose exceeded $800 \mathrm{mg} / \mathrm{dl}$. In $\mathrm{F}_{2}$, however, 24 of the 116 male rats and 14 of the 88 female rats had the sum of blood glucose exceeding $800 \mathrm{mg} / \mathrm{dl}$ and few of them exceeded $1000 \mathrm{mg} / \mathrm{dl}$. The distribution curves of the sum of blood glucose in $F_{2}$ are non-symmetrical but steep in left and gradual in right, i.e., a curve tailed to right. This seems to show the effect of the selective breeding.

Comment. This experiment demonstrated that offsprings of the rats with a slight impairment of glucose tolerance had a more impaired tolerance for glucose and some of the second generation had a diabetic GTT although they had no classical symptoms of diabetes 
such as polyuria and polydipsia. For the explanation of the present result, the multifactorial inheritance theory seems more reasonable than the theory assuming special diabetes gene(s). The further succession of this study will give many informations on natural history of diabetes including prediabetic stage, prevention of the development of diabetes and of the vascular complications, and so on.

Summary. The present study was undertaken to see the possibility of production of spontaneous diabetes rat by repeating the selective breeding of normal rats with a slight impairment of glucose tolerance. One hundred and thirty male and 81 female normal rats of Wistar strain were obtained from an experimental animal farm as foundation stock and 9 male and 9 female rats were chosen as breeding stock by oral glucose tolerance test. By the crossing, 89 male and 73 female rats were obtained $\left(F_{1}\right)$. The mean glucose tolerance curve of these offsprings was significantly higher than that of the foundation stock. Twelve male and 13 female rats were chosen among the $\mathrm{F}_{1}$ rats as breeding stock by glucose tolerance test. The crossing of them yielded 116 male and 88 female $\operatorname{rats}\left(\mathrm{F}_{2}\right)$. The mean glucose tolerance curve of $F_{2}$ was significantly higher than that of $F_{1}$ and some of them had a diabetic test and a positive test for urine sugar during the test. The result demonstrated the possibility of production of spontaneous diabetes from normal rats by the selective breeding.

\section{References}

1) Pincus, G., and White, P.: Amer. J. med. Sci., 186, 1 (1933).

2) Steinberg, A. G.: Diabetes, 10, 269 (1961).

3) Cammidge, P. J.: Brit. med. J., 2, 738 (1928); Lancet, 1, 393 (1934).

4) Stimmler, L., and Elliott, R. B.: Lancet, 1, 956 (1964).

5) Simpson, N. E.: Diabetes, 13, 462 (1964).

6) Craighead, J. E., and McLane, M. F.: Science, 162, 913 (1968). 